\begin{tabular}{lll}
\hline INTERNATIONAL & International Journal of Health Services Research and Policy \\
ENGINEERING, & (2019) 4(1): 22-30 \\
SCIENCE AND & Published online April, 2019 (http://dergipark.gov.tr/ijhsrp) \\
EDUCATION & doi: 10.23884/ijhsrp.2019.4.1.03 \\
GROUP & e-ISSN: 2602-3482 \\
& & Received: December 15, 2018 Accepted: March 26, 2019 \\
& & Submission Type: Research Article
\end{tabular}

\title{
HOSPITALIZATION RATES OF PATIENTS USING COMMUNITY MENTAL HEALTH CENTER SERVICES Şengül Kocamer Şahin ${ }^{1}$, Gülçin Elboğa ${ }^{1}$ Abdurrahman Altindag ${ }^{1}$
}

ORCID: 000000025371 3907; ORCID: 000000033903 1835; ORCID: 0000000155314419

${ }^{1}$ Gaziantep University Faculty of Medicine, Department of Psychiatry, Gaziantep

*Corresponding author; snglkcmr@hotmail.com

\begin{abstract}
Non-adherence to treatment and hospital admissions are the common problems in severe mental disorders. To determine the contribution of community mental health services to increased hospitalization rates of patients previously diagnosed with severe mental disorders such as schizophrenia spectrum and other psychotic disorders and bipolar disorders. 356 patients diagnosed with bipolar disorder, schizophrenia, schizoaffective disorder, and unspecified schizophrenia spectrum and other (USSO) psychotic disorders in accordance with the DSM-5 criteria and treated in the CMHC on the south coast of Turkey were included in this retrospective study.The hospitalization records of the patients were examined over three different periods. First and second period: two divided years which patients had not yet received CMHC services before registration. Third period: one-year, during which patients using CMHC services. A total of 356 patients were included in the study. SPSS 22.0 (IBM Corporation, Armonk, New York, United States) software was used in the analysis of variables. Normal distribution of data was assessed with the Shapiro-Wilk test. The diagnosis of the participants in the study were 157 (44\%) schizophrenia, 35 (9.8\%) schizoaffective disorder, 102 (28.7\%) bipolar disorder and $62(17.4 \%)$ unspecified schizophrenia spectrum and other psychotic disorders. As a result of the hospitalization rates, there was a significant difference between three periods. The median number of hospitalization days during the first episod was calculated as 1.0 (mean 13.9), the second period 0.7 (mean 18.59) and third period 0.6 (mean 5.83), respectively.The rate of hospitalization was $32.9 \%$ in the first period, $42.4 \%$ in the second period and $12.9 \%$ in the third period. This study provides the new evidence regarding the ability of primary services offered in the community mental health centers.
\end{abstract}

Key words: hospitalization, schizophrenia, bipolar, psychosis, community mental health center

\section{Introduction}

Non-adherence to treatment and hospital admissions are the common problems in the management of schizophrenia spectrum and related disorders, and bipolar disorders [1-3]. Reducing rehospitalisations in mental health care is a major challenge in Turkey. Rehospitalization is predicted by a combination of variables including the medication characteristics/adherence and healthcare- 
professional-related assessment [3,4]. Psychosocial interventions in psychiatric disorders may have long-term effects on recurrence and rehospitalisations rates [5]. In this context, educational programs are being developed for both patients, and the knowledge and attitudes of the patients about mental healthcare are reviewed [6]. An appointment reminder and coordination by a member of the health care team is likely to improve the treatment compliance with multidimensional implementation strategies such as educational materials or meetings [6]. A meta-analysis on psychoeducation has shown that if both patients and their family participate in psychoeducation, recurrence and re-admission rates decrease [7]. Additional treatment options would be useful to decrease recurrence and re-admission rates due to the fact that medical treatment alone is not sufficient. For this purpose, the World Health Organization (WHO) recommends the community-based mental health model (unlike hospital-based care) [8]. The development of Community Mental Health Centers with outpatient clinics in Turkey was one of the main strategies in a 2008 national action plan on mental health. Community mental health centers were established in Turkey to provide psychosocial support services for patients with severe mental illnesses such as bipolar disorder, schizophrenia spectrum and other psychoses [9]. The first study was conducted in Bolu CMHC (five years after the implementation of CMHC on a national plan for mental health in Turkey) on 18 patients with severe mental illness who were frequently admitted (more than three hospitalization in 18 months) to a hospital. The study showed that CMHC systems may reduce the number of acute hospitalizations [10]. However, as far as we know, there is no large-scale study in the literature about rehospitalization rates of all patients using CMHC services, regardless of participation frequency in Turkey.

We aimed to determine the contribution of community mental health services to increased hospitalization rates of patients previously diagnosed with severe mental disorders such as schizophrenia spectrum and other psychotic and bipolar disorders.

\section{Material and Method}

356 patients diagnosed with bipolar disorder, schizophrenia, schizoaffective disorder, and unspecified schizophrenia spectrum and other psychotic disorder (USS \& OPD) according to the DSM$\mathrm{V}$ criteria as assessed by psychiatrist and treated in the CMHC on the South coast of Turkey between 01 October 2016 and 31 September 2017 were included in this retrospective study. To evaluate the effectiveness of CMHC on hospitalization rates were compared taking into consideration three different periods: $1^{\text {st }}$ and $2^{\text {nd }}$ : Two years which patients had not yet received CMHC services and benefited from a standard care only before registration. $3^{\text {rd }}$ the one-year period, during which patients using CMHC services and received a standard clinic-based care. For example; if patient registrated CMHC on 01.11.2016, first period is determined as between 01.11.2014-.01.11.2015, second period 01.11.201501.11.2016, third period 01.11.2016-01.11.2017. The previous two-year period, during which patients had not yet received CMHC services, is determined as the first and second periods respectively to see the natural course of disorders without additional psychosocial interventions.

In the XXXX CMHC where the study was conducted, the patients were subjected to the psychoeducation process for at least every 6 months. Moreover, psychoeducation was provided in the home environment by visiting patients at home. The frequency of participation of the patients was determined by a phychiatrist and a psychologist according to the severity of the disease and the patient's preference. The patients using CMHC every three months or more frequently participated in the collective social activities at CMHC. The patients using CMHC every month or more frequently participated in daily skill therapies at CMHC. The patients using CMHC every week participated in 
group therapies at CMHC. With the appropriate schedules specific to the severity of illness, all patients were contacted at least every three months and the patients were provided with CMHC services by reminding via phone calls.

This study was conducted using routinely collected clinical data from CMHC. Information about the number of hospitalizations and a hospital name at the time of registration were obtained from patients and their family. During the one-year follow-up, new hospitalizations and hospital names were recorded. The information about hospitalization was scanned with the permission of presidency of public hospital services.

The patient exclusion criteria were as follows: being under 18 years of age, dementia, moderate or severe mental retardation, organic mental disorder and first-episode psychosis. The patients gave their consent, and the approval for the study was obtained from the Medical Ethics Committees of the institutions.

Days spent in a hospital were retrospectively screened. These assessment score were compared for three periods.

\subsection{Statistical Analysis}

SPSS 22.0 (IBM Corporation, Armonk, New York, United States) software was used in the analysis of variables. Normal distribution of data was assessed with the Shapiro-Wilk test. MannWhitney U Test was used with Monte Carlo results to compare two independent groups with each other according to quantitative data. In order to compare the categorical variables with each other, the Pearson Chi-Square, Fisher Exact and Fisher-Freeman-Holton tests were used and provided with Monte Carlo and Exact results. The Kendall's tau-b test was used to analyze the correlations between the variables. The quantitative variables were expressed as median range (maximum-minimum) and categorical variables as $\mathrm{n}(\%)$. The variables were analyzed at a confidence interval of $95 \%$ and a $\mathrm{p}$ value of $<0.05$ was accepted as statistically significant.

\section{Results}

A total of 356 patients were included in the study: 157 (44\%) schizophrenia, 35 (9.8\%) schizoaffective disorder, $102(28.7 \%)$ bipolar disorder and 62 (17.4\%) unspecified schizophrenia spectrum and other psychotic disorder (USS \& OPD) patients. The mean age of the patients was $41.1 \pm 12.5$ years, the mean age of onset of illness was $23.4 \pm 9.1$ years and the mean number of life-long

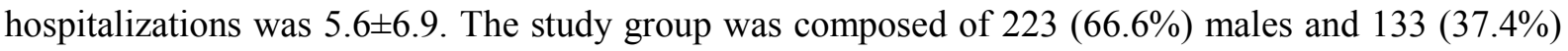
females. The sociodemographic data of the patients are shown in Table 1. 
Table 1. The sociodemographic data of the patients

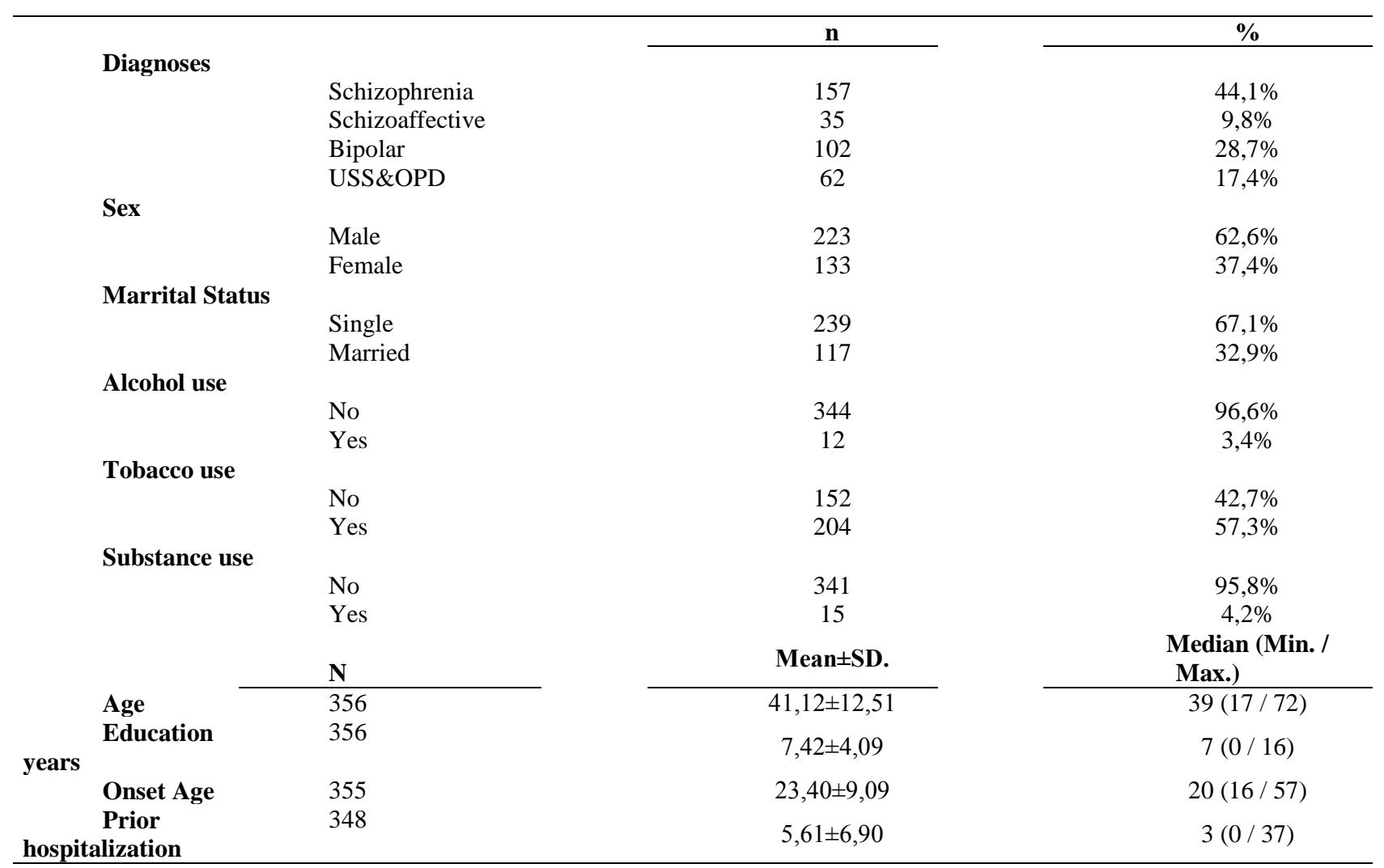

SD.:Standard deviation, Min.:Minimum, Max.:Maximum

The median number of days spent in a hospital during the first period was calculated as 1.0 (mean 13.9), the second period 0.7 (mean 18.59) and during the third period 0.6 (mean 5.83), respectively. There were not significant differences between the first and second period. There were significant differences between the third period and first/second period (Table 2). The total number of days spent in a hospital during the first period was calculated as 4730, the second period 6258 and during the third period 2047 days, respectively.

Since the data did not comply with the normal distribution, hospitalizations were also evaluated as hospitalization rates of each period. The hospitalization rate during the first period was $32.9 \%$, during the second period was $42.4 \%$ and during the third period was $12.9 \%$. There were significant differences between three periods (Table 2). 
Table 2. The number of days spent in hospital and hospitalization rates

\begin{tabular}{|c|c|c|c|c|c|c|c|}
\hline \multirow{2}{*}{ TOTAL } & \multirow{2}{*}{ First period } & \multirow{2}{*}{ Second Period } & \multirow{2}{*}{ Third Period } & \multirow{2}{*}{$\mathbf{P}$} & \multicolumn{3}{|c|}{ Binary Comparisons } \\
\hline & & & & & $\mathbf{P}(\mathbf{1 - 2})$ & $\mathbf{P}(\mathbf{1 - 3})$ & $\mathbf{P}(2-3)$ \\
\hline & $\begin{array}{r}\text { Median } \\
\text { (Min./Max.) }\end{array}$ & $\begin{array}{r}\text { Median } \\
\text { (Min./Max.) }\end{array}$ & $\begin{array}{c}\text { Median } \\
\text { (Min./Max.) } \\
\end{array}$ & & & & \\
\hline \multirow[t]{2}{*}{$\begin{array}{l}\text { Number of days } \\
\text { spent in hospital }\end{array}$} & $1,0(0 / 186)$ & $0,7(0 / 265)$ & 0,6 (0 / 196) & $<0,001$ & 0,242 & $<0,001$ & $<0,001$ \\
\hline & n $(\%)$ & n $(\%)$ & n $(\%)$ & & & & \\
\hline \multicolumn{8}{|l|}{ Hospitalization } \\
\hline $\begin{array}{l}\text { No } \\
\text { Yes }\end{array}$ & $\begin{array}{l}239(67,1) \\
117(32,9)\end{array}$ & $\begin{array}{l}205(57,6) \\
151(42,4)\end{array}$ & $\begin{array}{c}310(87,1) \\
46(12,9)\end{array}$ & $<0,001$ & 0,002 & $<0,001$ & $<0,001$ \\
\hline
\end{tabular}

Cochran's Q test(Monte Carlo), Friedman Test(Monte Carlo); Post Hoc Test : Dunn's Test, Min.:Minumum Max.:Maximum

There were statistically significant difference between bipolar disorder and USS \& OPD patients when comparing the first and second periods. There was statistically significant difference between schizophrenia and USS \& OPD patients when comparing the first and second periods (Table 3).

There was no statistically significant difference between all diagnostic groups when comparing the second and third periods, and the first and third periods with all assessment scores (Table 3).

Table 3. Comparison between diagnostic groups in terms of hospitalized days

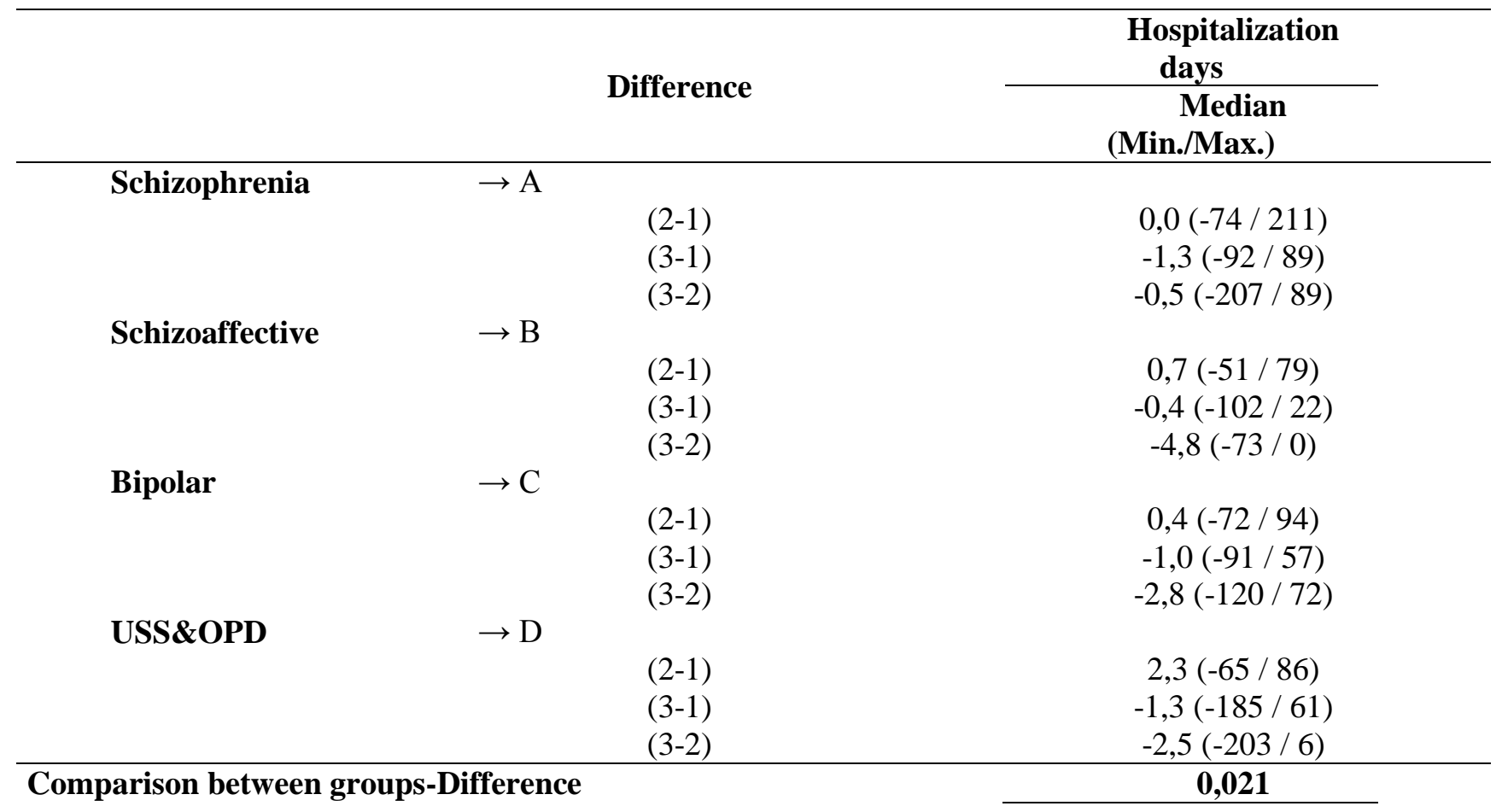


$(2-1)$

\begin{tabular}{cc}
$\mathbf{A} \rightarrow \mathbf{B}$ & 0,103 \\
$\mathbf{A} \rightarrow \mathbf{C}$ & 0,529 \\
$\mathbf{A} \rightarrow \mathbf{D}$ & $\mathbf{0 , 0 0 4}$ \\
$\mathbf{B} \rightarrow \mathbf{C}$ & 0,251 \\
$\mathbf{B} \rightarrow \mathbf{D}$ & 0,559 \\
$\mathbf{C} \rightarrow \mathbf{D}$ & $\mathbf{0 , 0 3 1}$ \\
\hline $\begin{array}{c}\text { Comparison between groups-Difference } \\
(3-1)\end{array}$ & 0,538 \\
\hline $\begin{array}{c}\text { Comparison between groups-Difference } \\
(3-2)\end{array}$ & 0,403
\end{tabular}

Kruskal Wallis Test(Monte Carlo); Post Hoc Test : Dunn's Test, Min.:Minumum Max.:Maximum

There were not significant differences between the first and second period in terms of hospitalization days in schizophrenia patients but there were significant differences between third period and first/second period (Table 4).

Table 4. The number of days spent in hospital for each diagnostic groups

\begin{tabular}{|c|c|c|c|c|}
\hline \multirow[b]{2}{*}{ Diagnosis } & \multirow[b]{2}{*}{ Peryot } & \multirow{2}{*}{$\begin{array}{c}\text { Hospitalization days } \\
\text { Median } \\
\text { (Min./Max.) }\end{array}$} & \multirow{2}{*}{$\begin{array}{l}\text { Hospitalization- } \\
\text { No } \\
\qquad \begin{array}{l}\text { n }(\%) \\
\end{array}\end{array}$} & \multirow{2}{*}{$\begin{array}{l}\text { Hospitalization- } \\
\text { Yes } \\
\text { n (\%) }\end{array}$} \\
\hline & & & & \\
\hline \multirow[t]{4}{*}{ Schizophrenia } & $\rightarrow \mathrm{A}$ & & & \\
\hline & 1 & $1,9(0 / 146)$ & $(68,2)^{107}$ & $(31,8)^{50}$ \\
\hline & 2 & $0,6(0 / 211)$ & $(59,9)^{94}$ & $(40,1)^{63}$ \\
\hline & 3 & $0,6(0 / 119)$ & $(87,3)^{137}$ & $(12,7)^{20}$ \\
\hline \multirow[t]{4}{*}{ Schizoaffective } & $\rightarrow \mathrm{B}$ & & & \\
\hline & 1 & $0,8(0 / 186)$ & $(71,4)^{25}$ & $(28,6)^{10}$ \\
\hline & 2 & $4,8(0 / 265)$ & $(57,1)^{20}$ & $(42,9)^{15}$ \\
\hline & 3 & $2,0(0 / 196)$ & $(82,9)^{29}$ & $6(17,1)$ \\
\hline \multirow[t]{4}{*}{ Bipolar } & $\rightarrow \mathrm{C}$ & & & \\
\hline & 1 & $1,3(0 / 115)$ & $(60,8)^{62}$ & $(39,2)^{40}$ \\
\hline & 2 & $4,1(0 / 153)$ & $(58,8)^{60}$ & $(41,2)^{42}$ \\
\hline & 3 & $1,1(0 / 94)$ & $(86,3)^{88}$ & $(13,7)^{14}$ \\
\hline \multirow[t]{4}{*}{ USS \% OPD } & $\rightarrow \mathrm{D}$ & & & \\
\hline & 1 & $1,8(0 / 185)$ & $(72,6)^{45}$ & $(27,4)^{17}$ \\
\hline & 2 & $4,4(0 / 203)$ & $(50,0)^{31}$ & $(50,0)^{31}$ \\
\hline & 3 & $0,6(0 / 117)$ & $(90,3)^{56}$ & $6(9,7)$ \\
\hline \multicolumn{2}{|c|}{ Schizophrenia Group P Value } & $<0,001$ & \multicolumn{2}{|r|}{$<\mathbf{0 , 0 0 1}$} \\
\hline & $1 \rightarrow 2$ & 1 & \multicolumn{2}{|r|}{0,105} \\
\hline & $1 \rightarrow 3$ & 0,002 & \multicolumn{2}{|r|}{$<0,001$} \\
\hline & $2 \rightarrow 3$ & 0,001 & \multicolumn{2}{|r|}{$<0,001$} \\
\hline
\end{tabular}




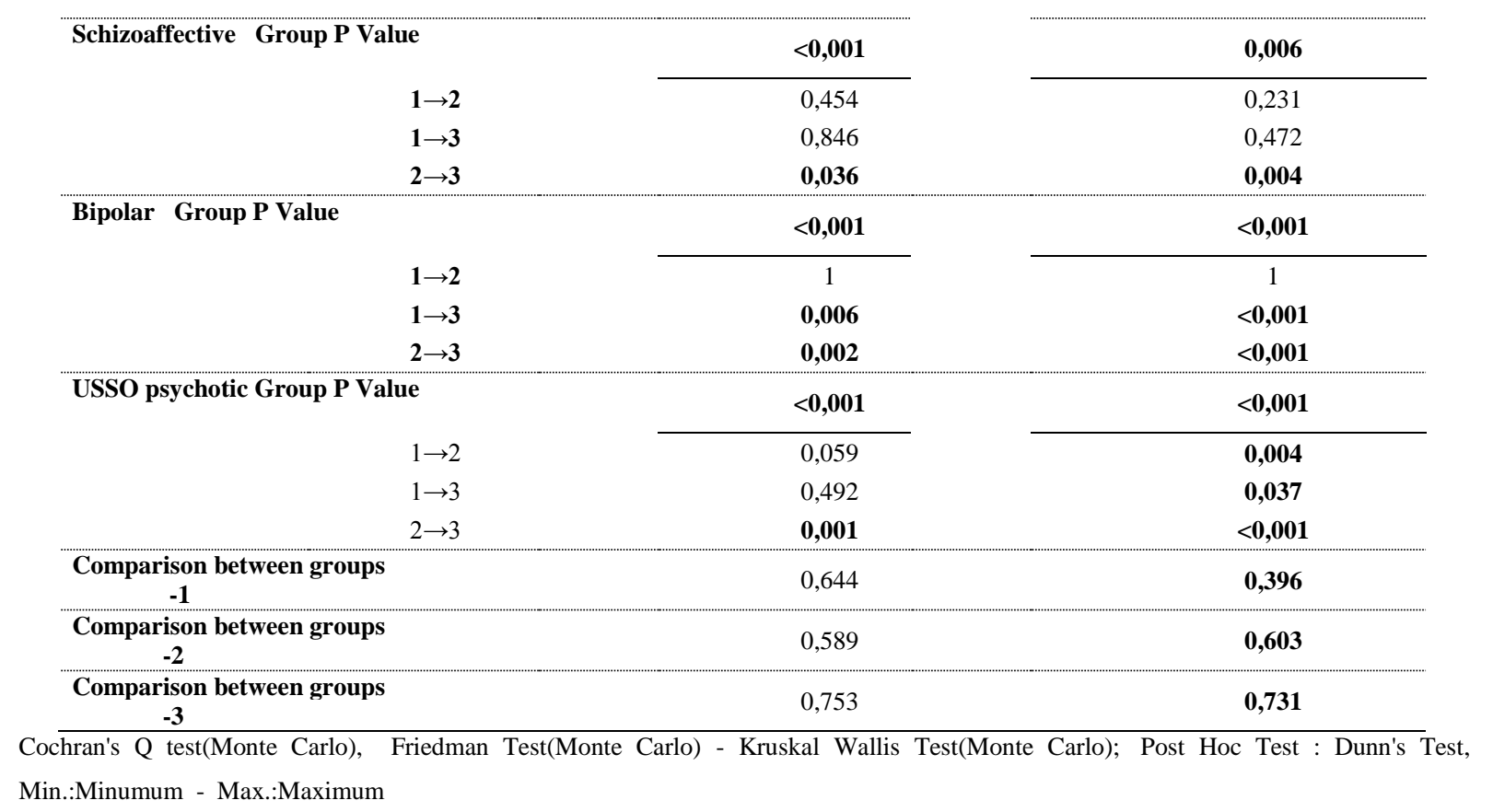

There were not significant differences between the first and second/third period in terms of hospitalization days in patients with schizoaffective disorder; however, there were significant differences between the second and third periods (Table 4).

There were not significant differences between the first and second periods in terms hospitalization days in bipolar patients; however, there were significant differences between the third period and first/second period (Table 4).

There were not significant differences between the first and third periods in terms of hospitalization rates in USS \& OPD patients. There were significant differences between the third and second period. There were significant differences between the first and second periods in terms of total number of days spent in a hospital (Table 4).

\section{Discussion}

This study investigated the association between CMHC services and hospitalization days in patients with schizophrenia spectrum and other psychosis and bipolar disorder patients. The participation in the CMHC was observed to be a positive contribution to illness management taking into consideration rehospitalization rates.

Firstly, we found that the rate of hospitalizations significantly increased during the second period when patients had not yet received the CMHC services during the natural course of the disease. In the natural course of the diseases, the diagnosis of bipolar disorder was found to be in lower relative risk as 0.12 than schizophrenia and other psychosis in terms of rehospitalizations [11]. In our study we found significant differences between the first and second periods in only USS \& OPD patients when we assessed each category of diagnosis.

It was noteworthy that the number of days spent in hospital decreased after patients participated in the CMHC. The early intervention services were found to be associated with better outcomes than treatment as usual [11]. We found that community mental health centers achieve decrease in rehospitalization in adults with chronic mental disorders. This study shows that psychosocial interventions are still effective even in later stages of mental disorders. 
In other studies, physicians stressed treatment adherence effects on rehospitalization rates $[1,2$, 4]. Non-adherence to treatment increases the hospital admission $[1,12]$. However, rehospitalization is predicted not only by treatment adherence but also by a combination of variables such as medication characteristics and healthcare-professional-rated assessment ${ }^{3}$. In our study, it was noteworthy that using CMHC services on a regular basis and multidimensional implementation strategies such as educational materials, treatment monitoring, family education, group therapies, organization of both in-and outpatient mental health services, easy access to the medical team even via phone or by outpatient visits, significantly decreases rehospitalization rates. All of these strategies should provide a positive contribution to the illness management and decrease in rehospitalization rates [13-18]. Such strategies must be implemented in typical real-world settings.

A limitation of our study was the lack of a prospective study and a control group consisting of patients benefited only from outpatient clinic services. Significant heterogenity in diagnosis of disorders and only one-year follow-up is another limitation of this study.

\section{Conclusion}

This study provides the new evidence regarding the ability of primary services offered in community mental health centers to achieve decrease in rehospitalization in adults with chronic mental health issues.

\section{Acknowledgements}

The authors acknowledge Adana Public Hospitals Services President Gorgulu O, C.Y. Ahmet, Tulu G, Hayta S and Community Mental Health Center team; Yumusak MM, Gonul N, Kuruoglu H, Boz A for assistance with collection of data.

\section{References}

[1]. Boden, R et al. "Early non-adherence to medication and other risk factors for rehospitalization in schizophrenia and schizoaffective disorder", Schizophrenia Research,133, 36-41, 2011.

[2]. MacDonald, L et al., "Improving medication adherence in bipolar disorder: A systematic review and meta-analysis of 30 years of intervention trials", J Affect Disord,194, 202-221, 2016.

[3]. Koolmees, AH et al., "Predicting rehospitalization in patients treated with antipsychotics: A prospective observational study", Ther Adv Psychopharmacol, 8, 213-229. 2018.

[4]. Shao, WC et al., "The relationship between medication adherence and rehospitalization: A prospective study of schizophrenia patients discharged from psychiatric acute wards", $\mathrm{Hu} \mathrm{Li} \mathrm{Za}$ Zhi, 60, 31-40, 2013.

[5]. Guo, X et al., "Effect of antipsychotic medication alone vs combined with psychosocial intervention on outcomes of early-stage schizophrenia: A randomized, 1-year study", Arch gen psychiatry, 67: 895-904, 2010.

[6]. Girlanda, F et al., "Guideline implementation strategies for specialist mental healthcare", Curr Opin Psychiatry, 26, 369-375, 2013.

[7]. Sin J, Gillard S, Spain D et al., "Effectiveness of psychoeducational interventions for family careers of people with psychosis: A systematic review and meta-analysis", Clin Psychol Rev, 56: 13-24, 2017.

[8]. Organization WH . The World Health Report, Mental health: New understanding, new hope. World Health Organization. 2001 
[9]. Ensari, $\mathrm{H}$ et al., "The effects of the service of community mental health center on the schizophrenia patients -evaluation of quality of life, disabilities, general and social functioning a summary of one year follow-up", Anatolian J Psychiatry, 14, 108-214, 2013

[10].Aydın, E et al., "Revolving door phenomenon: The effect of application of community-based mental health services model at acute psychiatric hospitalization”, Anatolian J Psychiatry, 15,185-191, 2014.

[11]. Correll, CU et al., "Comparison of Early Intervention Services vs Treatment as Usual for Early-Phase Psychosis: A Systematic Review, Meta-analysis, and Meta-regression", JAMA Psychiatry, 1, 555-565, 2018.

[12]. Ucok, A et al., "One year outcome in first episode schizophrenia: Predictors of relapse", Eur Arch Psychiatry Clin Neurosci,256, 37-43, 2006

[13]. Grinshpoon, A et al., "Post-discharge contact with mental health clinics and psychiatric readmission: A 6-month follow-up study", Isr J Psychiatry Relat Sci,48, 262-267, 2011.

[14]. Lee, SY et al., "Outpatient Follow-Up Visit after Hospital Discharge Lowers Risk of Rehospitalization in Patients with Schizophrenia: A Nationwide Population-Based Study", Psychiatry Investig, 12, 425433, 2015.

[15]. Swain, SP \& Behura SS., "A comparative study of quality of life and disability among schizophrenia and obsessive-compulsive disorder patients in remission”, Ind psychiatry J, 25 , 210-215, 2016.

[16].Haddad, PM et al., "Nonadherence with antipsychotic medication in schizophrenia: Challenges and management strategies", Patient Relat Outcome Meas, 5, 43-62, 2014.

[17]. Sims, H et al., "Text message reminders of appointments: A pilot intervention at four community mental health clinics in London”, Psychiatr Serv, 63, 11961-1968, 2012.

[18].Schöttle, D et al., "Effectiveness of integrated care including therapeutic assertive community treatment in severe schizophrenia-spectrum and bipolar I disorders: Four-year follow-up of the ACCESS II study”, PLoS One, 13, e0192929, 2018. 